\title{
Twenty Challenging Minutes to Take the Right Decision
}

\author{
Dr. Priyanka P Shetty ${ }^{1}$, Dr Ramadas Naik ${ }^{2}$, Dr Vineeth G Nair ${ }^{3}$, Dr Shubha Sudhakar ${ }^{4}$, \\ Dr Ali Abdul Latheef ${ }^{5}$ \\ ${ }^{1}$ Junior Resident, Department of Pathology, Yenepoya Medical College, Mangaluru, India \\ ${ }^{2}$ Dr Ramadas Naik- Professor and Head of Department, Department of Pathology, Yenepoya Medical College, \\ Mangaluru, India \\ ${ }^{3}$ Pathologist, Suburban Diagnostics, North Goa \\ ${ }^{4}$ Assistant Professor, Department of Pathology, Yenepoya Medical College, Mangaluru India \\ ${ }^{5}$ Junior resident, Department of Pathology, Yenepoya Medical College, Mangaluru, India
}

*Corresponding author: Dr Priyanka P Shetty, priyankashetty112@gmail.com

Received 12 February 2019;

Accepted 07 March 2019;

Published 12 March 2019

\begin{abstract}
Background: The diagnostic accuracy of frozen section is an important source of information and a high risk procedure in surgical pathology. This is an important tool, not only in the management of surgical patients but also has a pivotal role in institution's quality assurance in histopathology. Aims and objectives: To analyze indications, efficacy, accuracy, limitations, deferrals and turnaround time for all frozen sections in correlation with histopathology. Materials and methods: A retrospective analysis of all the frozen samples was done in correlation with permanent sections from June 2016 till July 2017. The diagnostic accuracy, sensitivity, specificity, turnaround time, deferrals and limitations were analysed. Results: A total of 45 specimens for frozen sections were sent to the department of Pathology during the time period. Out of these, $97.8 \%(n=44)$ were accepted and $2.2 \%(n=1)$ were deferred. Of the remaining 44 specimens, $1(2.3 \%)$ was discordant and 43 (97.7\%) were concordant to permanent diagnoses. Specimens were primarily from the ovary, breast and lymph nodes. The overall sensitivity, specificity, positive predictive value and negative predictive value of the frozen section compared to formalin fixed paraffin embedded sections (as gold standard) were $97.7 \%, 100 \%, 100 \%$ and $100 \%$ respectively. The average turn-around time for frozen section diagnosis was 23 minutes. Conclusion: Frozen section diagnosis is an efficient, rapid, reliable and highly accurate tool in surgical diagnosis and management. The multidisciplinary approach helped avoiding limitations ensuring optimal patient care.
\end{abstract}

Keywords: Frozen section, Histopathology, Sensitivity, Specificity, Accuracy, Quality assurance, Turnaround time

\section{Introduction}

Frozen section or cryotomy, is a method of cutting unfixed tissue. These frozen sections are used for immediate intraoperative diagnosis with the help of a surgical pathologist, and can also be used to perform enzyme histochemistry and immunohistochemistry. ${ }^{[1]}$ The frozen section technique was introduced by William $\mathrm{H}$ Welch and this was during a surgical procedure performed by surgeon William Halstead in John Hopkins Hospital in 1891; however, Halstead walked out the of the operation theatre before he received the report, therefore this was not the first instance of a frozen section being used to clinical advantage. Later the pathologist Ernest Shaw who was a pathologist at Great Northen Hospital in London in 1899 performed the test to completion. ${ }^{[2]}$ In 1905, Louis B Wilson at the Mayo Clinic developed the use of methylene blue for staining rapid frozen section. Due to the adoption of frozen sections there was a drop in the rate of inoperable cancers in Johns Hopkins from 50\% in 1905 to less than $5 \%$. $^{[3]}$

More than 100 years later, even with the advent of modern technology and numerous other advancements in the field of surgical pathology, frozen sections remain an important tool for both the surgeon and the surgical pathologists alike. Its importance is compounded by the fact that it also plays a pivotal role in an institution's quality assurance in histopathology.

\section{Aim}

The aim of this study was to analyse the indications, efficacy, accuracy, limitations, deferrals and turn-around time of intraoperative frozen sections. 


\section{Materials and Methods}

This was a retrospective study conducted at the Department of Pathology, Yenepoya Medical College, Mangalore. All cases of frozen sections done between June 2016 and July 2017 were analysed - a total of 45 cases.

The procedure for frozen sections in the institution for the time period mentioned was as follows:

1. A prior appointment for frozen section is taken the day prior to the surgery.

2. The specimen for frozen section analysis is sent from the operation theatre in saline along with the test requisition form.

3. The specimen is grossed and representative sections given.

4. The sections are placed in a freezing medium (cryostat OCT compound) on chucks.

5. Once the medium and tissue is frozen, the chuck is inserted in the clamping lever of the cryostat.

6. The cryostat is set between $-18{ }^{\circ} \mathrm{C}$ and $-24{ }^{\circ} \mathrm{C}$ and sections cut at a thickness of 5-6 microns and immediately fixed in isopropyl alcohol onto a glass slide.

7. Rapid haematoxylin and eosin (H\&E) staining is performed on these slides.

8. The slides are reviewed by experienced pathologists; a diagnosis is arrived at and then communicated to the surgeon immediately.

9. The remaining specimen is then immersed in $10 \%$ neutral buffered formalin for fixation.

10. After fixation, the specimen is re-grossed, sections given and embedded in paraffin.

11. Slides are prepared, stained with $H \& E$ and studied for histopathological features and a diagnosis is arrived at.

In every case, the concordance or discordance of the frozen section diagnosis was analysed with respective to the final histopathological diagnosis. The sensitivity, specificity, turnaround time, deferrals if any and limitations were also analysed.

\section{Results}

A total of 45 specimens for frozen sections were sent to the department of Pathology during the time period. Out of these, 97.8 $\%(\mathrm{n}=44)$ were accepted and $2.2 \%(\mathrm{n}=1)$ were deferred. The one case which was deferred was because the specimen was sent in formalin as opposed to saline.

The specimens were primarily from the ovary, breast and brain (Figure 1).

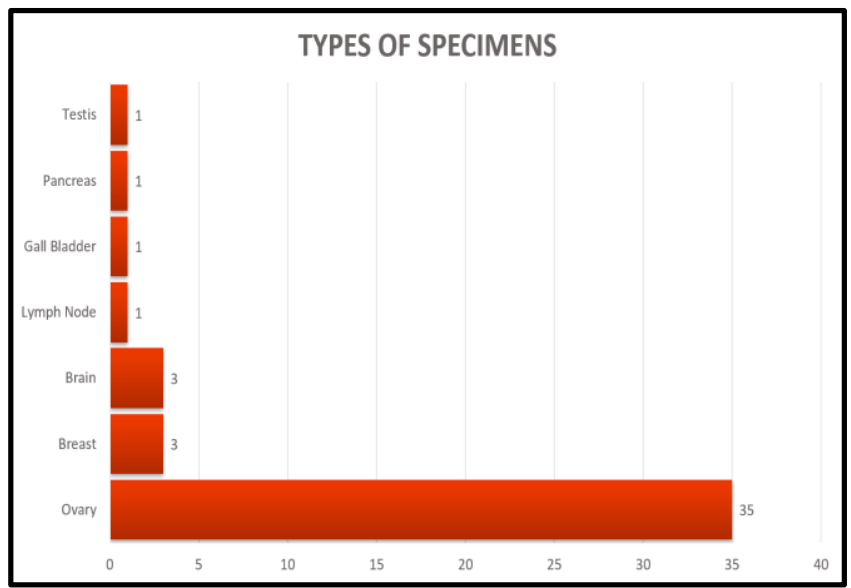

Figure 1- Types of Specimens Received

The highest number of specimens was from patients belonging to the age group of $40-49$, followed by the $30-39$ age groups (Figure 2).

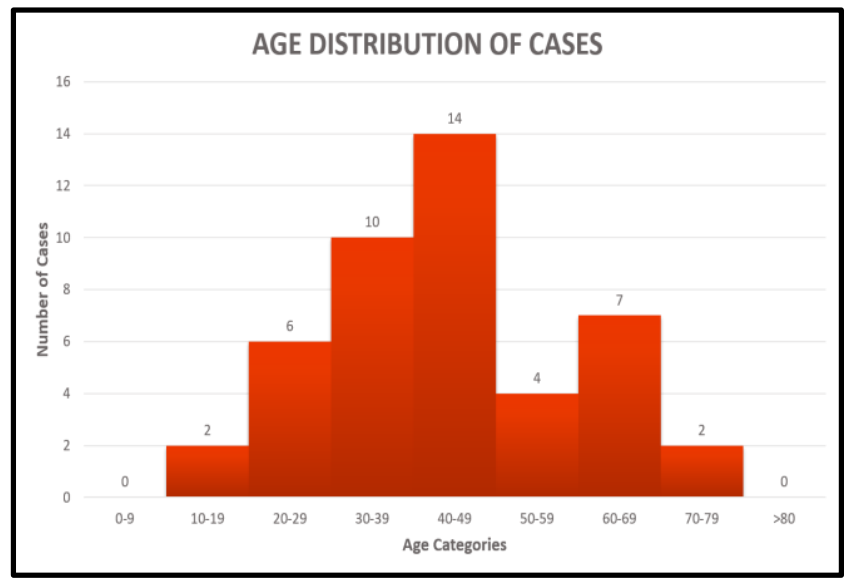

Figure 2: Age Distribution of Cases

Of the accepted specimens, $97.7 \% \quad(n=43)$ were found to be concordant with the final diagnosis and $2.3 \%(\mathrm{n}=1)$ discordant (Table 1).

Table 1 - Types, number and concordancy of cases

\begin{tabular}{|l|c|c|c|c|}
\hline \multicolumn{1}{|c|}{ Specimen } & Number of Cases & $\begin{array}{c}\text { Number of Concordant } \\
\text { Diagnoses }\end{array}$ & $\begin{array}{c}\text { Number of Discordant } \\
\text { Diagnoses }\end{array}$ & $\begin{array}{c}\text { Number of Deferred } \\
\text { Cases }\end{array}$ \\
\hline Ovary & 35 & 34 & 1 & 0 \\
\hline Breast & 3 & 3 & 0 & 0 \\
\hline Brain & 3 & 3 & 0 & 0 \\
\hline Lymph Node & 1 & 0 & 0 & 1 \\
\hline Gall Bladder & 1 & 1 & 0 & 0 \\
\hline Pancreas & 1 & 1 & 0 & 0 \\
\hline Testis & 1 & 1 & 0 & 0 \\
\hline TOTAL & $\mathbf{4 5}$ & $\mathbf{4 3}$ & $\mathbf{1}$ & \\
\hline
\end{tabular}

The one discordant case was found to be so, because the typicality was focal and wasn't sampled during the frozen section.

Amongst the 44 cases, 4 were sent for margin/lymph node status and the other 40 were sent for intraoperative diagnosis. Of these
40, 30 were found to be benign, 9 were malignant and 1 was diagnosed as atypical/borderline tumour.

The overall sensitivity, specificity, positive predictive value and negative predictive value of the frozen section compared to 
formalin fixed paraffin embedded sections (as gold standard) were $97.7 \%, 100 \%, 100 \%$ and $100 \%$ respectively.

As mentioned earlier, the indication in the majority of cases was to establish an intraoperative diagnosis and in a few cases, to assess margin/lymph node status. Some of the cases received during the study are show in figures 3-7. However we also encountered a few problems during the study such as cutting artefacts (fig 7) and staining artefacts (fig 6). In one of the cases (fig6) the frozen section showed gland and signet ring cells like arrangement. However this case was just diagnosed as a malignant tumour on frozen section and further history was asked for and finally diagnosed as a low grade malignant fibrothecoma.

The average turnaround time was found to be 23 minutes.

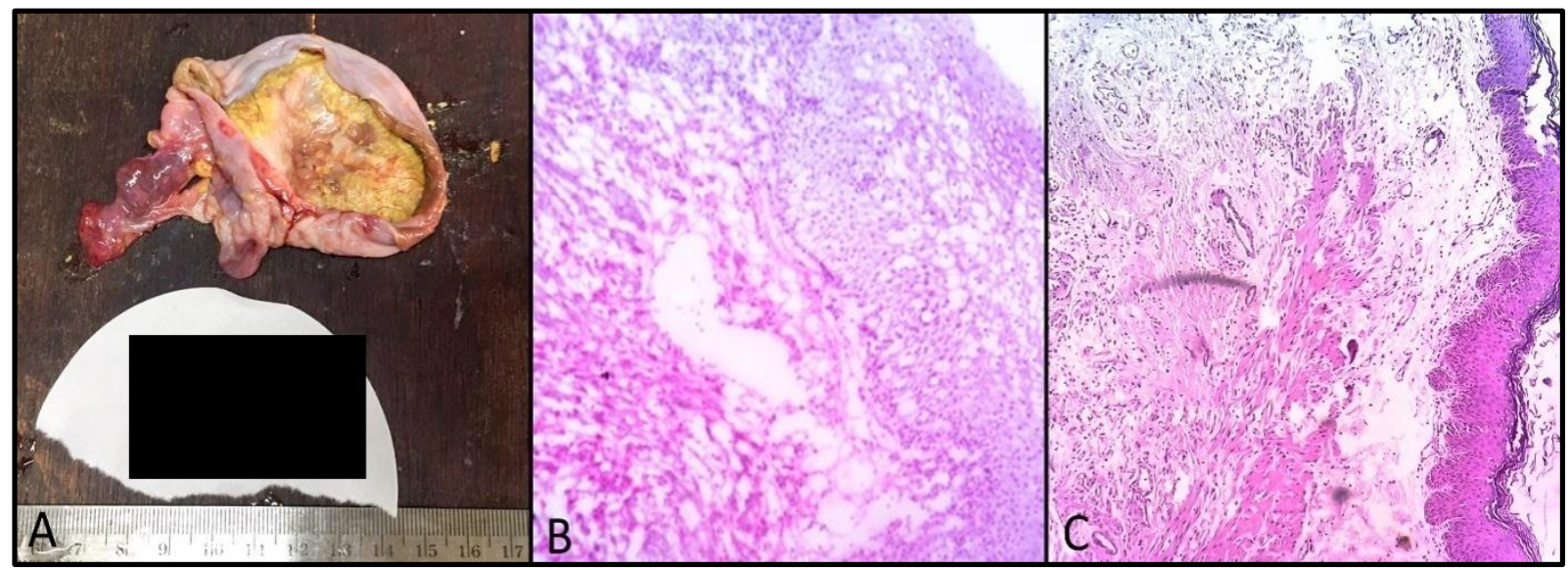

Figure 3: (A) Mucinous adenocarcinoma gross; (B) Photomicrograph of frozen section (2); (C) Photomicrograph of paraffin embedded section

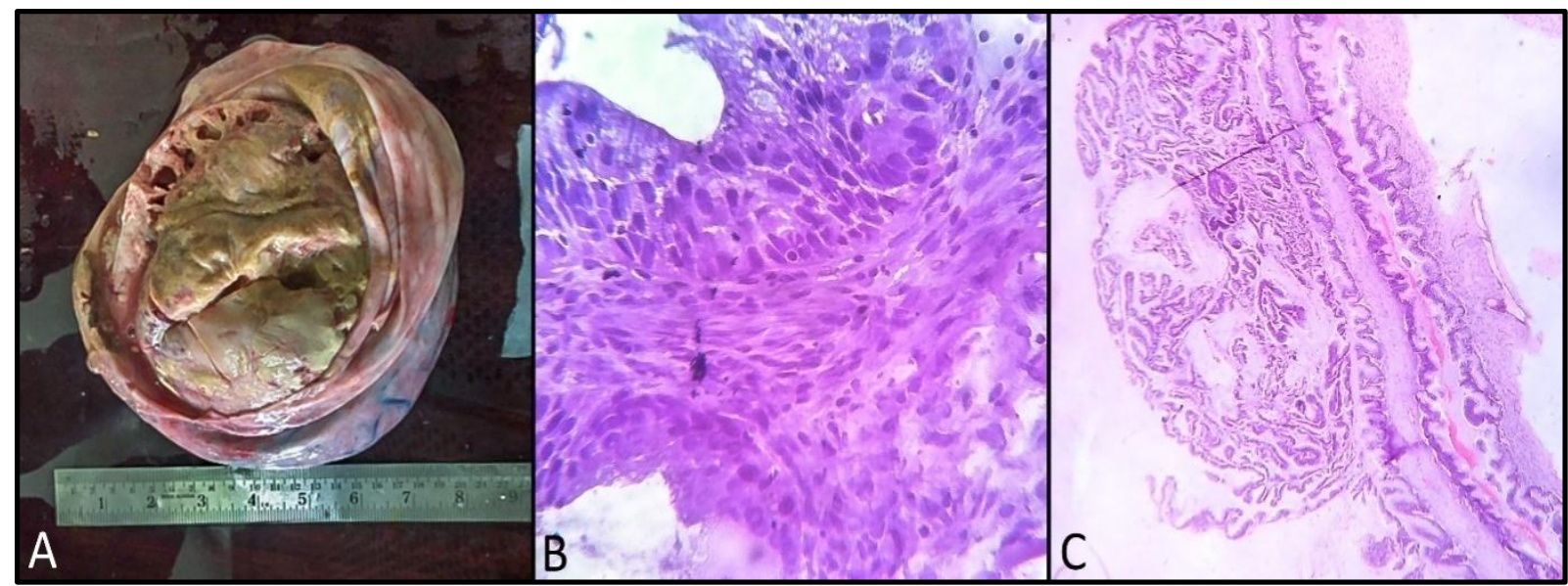

Figure 4: (A) Mucinous adenocarcinoma gross; (B) Photomicrograph of frozen section; (C) Photomicrograph of paraffin embedded section.

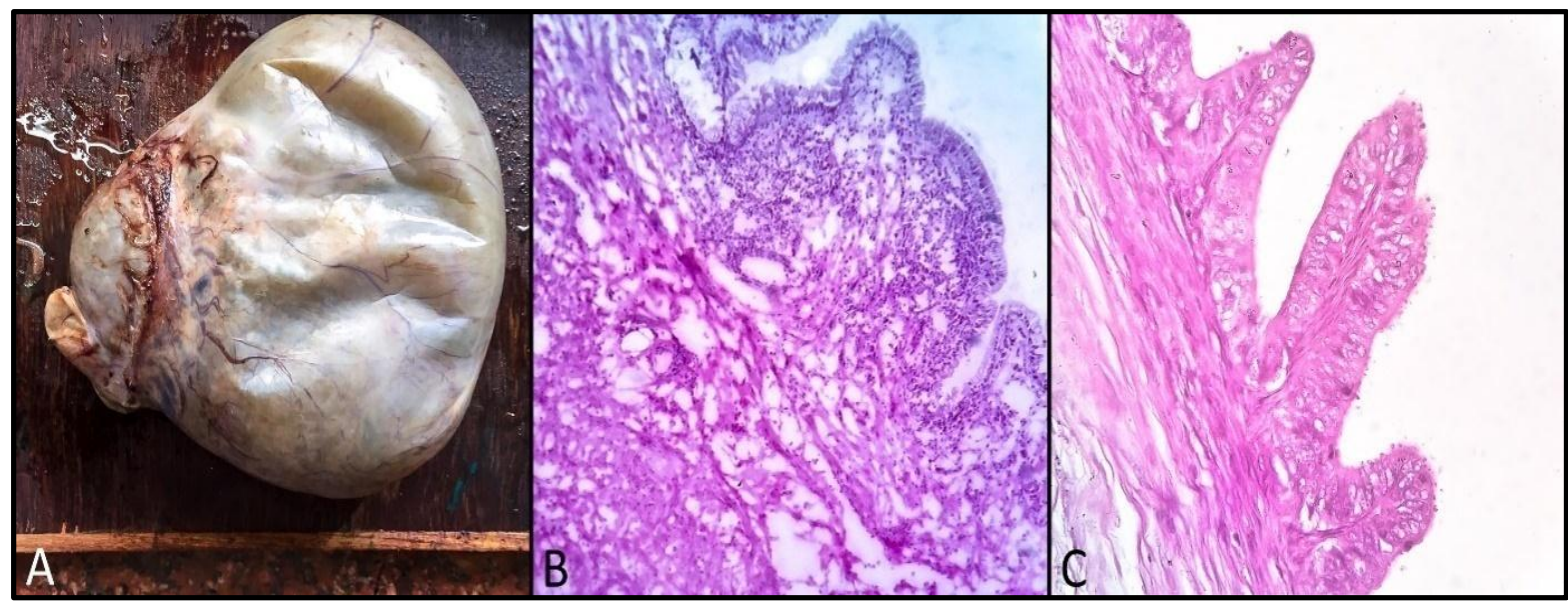

Figure 5: (A) Mucinous borderline tumour gross; (B) Photomicrograph of frozen section; (C) Photomicrograph of paraffin embedded section 


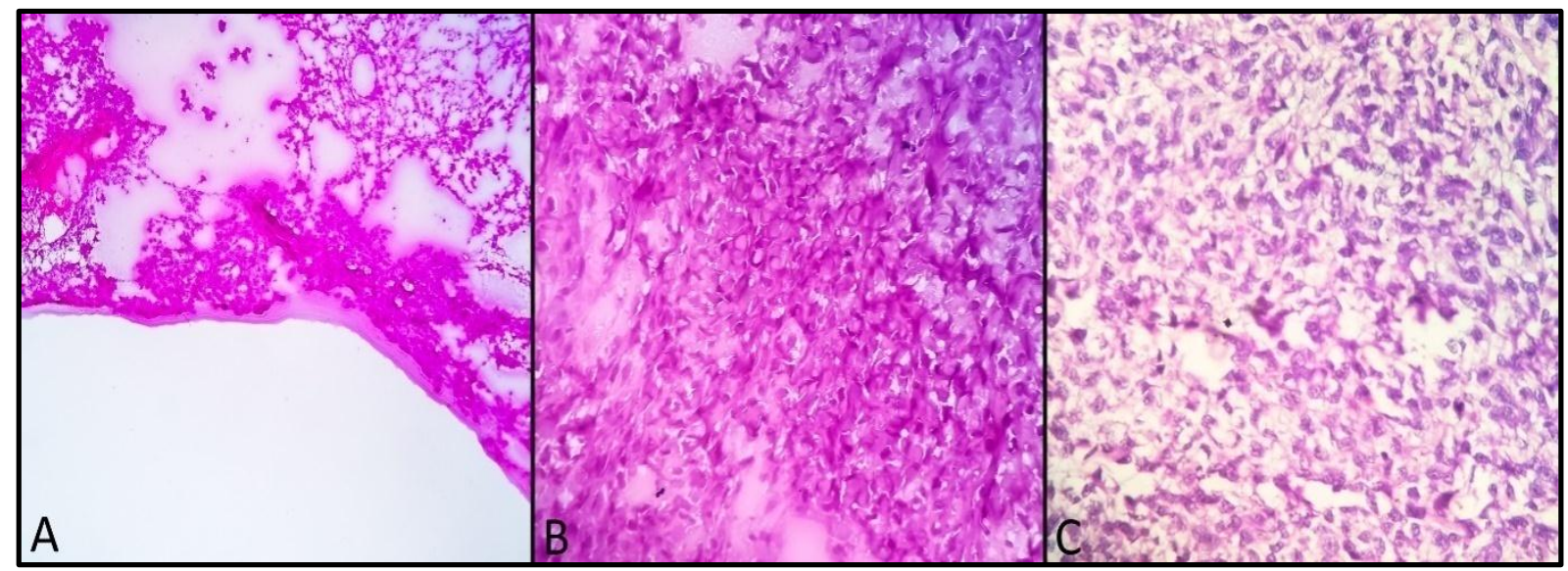

Figure 6: (A) Frozen Section - Tumour arranged in focal glands and papillary pattern; (B) Frozen Section - Areas showing signet ring cell like pattern; (C) Paraffin embedded section - final diagnosis was low grade malignant fibrothecoma.

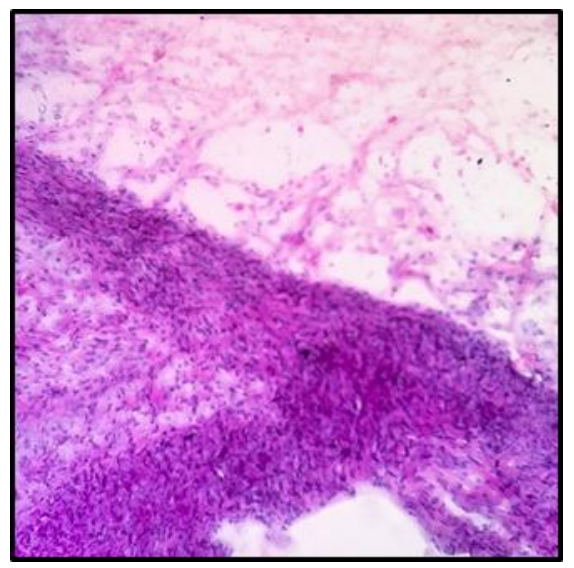

Figure 7: Occasional cases showed folding artefacts as above

\section{Discussion}

In the present study, we compared the diagnosis established in frozen section to that of paraffin embedded sections to determine the accuracy of frozen section diagnosis in histopathology. Ovarian tisuue was the predominant sample similar to the studies conducted by Saumya Mishra et al (2016). ${ }^{[4]}$ The overall sensitivity, specificity, positive predictive value and negative predictive value of the frozen section compared to formalin fixed paraffin embedded sections were $97.7 \%, 100 \%, 100 \%$ and $100 \%$ respectively. Studies conducted by Hossein Hatami et al (2015), ${ }^{[5]}$ Patil et al (2015), ${ }^{[6]}$ JunnLiang et al (1992) ${ }^{[7]}$ and Agarwal Preeti et al $(2016)^{[8]}$ showed similar results (Table 2). The overall accuracy was found to be as high as $99 \%$ in concordance with studies conducted by Saumya Mishra et al (2016), ${ }^{[4]}$ Agarwal Preeti et al (2016), ${ }^{[8]}$ Shresta et al (2009) ${ }^{[9]}$ and Patil et al (2015) ${ }^{[6]}$ (Table 3). One case was deferred in our study and 44/45 cases concordant with the histopathological diagnosis similar to study conducted by Chandramouleshwari et al (2013). ${ }^{[10]}$ The average turnaround time was 23 minutes similar to that in the study by Saumya Mishra et al $(2016)^{[4]}$ where it was 20 mins.

Table 2: Comparison of Sensitivity, Specificity, PPV and NPV to other studies

\begin{tabular}{|l|c|c|c|c|c|}
\hline Authors & Sensitivity & Specificity & PPV & NPV & Accuracy \\
\hline Hatami et al (2015) & $92.95 \%$ & $99.55 \%$ & $98.5 \%$ & $97.8 \%$ & - \\
\hline Patil et al (2015) $^{\mathbf{6}}$ & $97.23 \%$ & $96.30 \%$ & $98.59 \%$ & $92.8 \%$ & $96.96 \%$ \\
\hline JunnLiang et al (1992) $^{\mathbf{7}}$ & $96.16 \%$ & $99.43 \%$ & - & - & $97.7 \%$ \\
\hline Preeti et al (2016) & $\mathbf{8}$ & $97.5 \%$ & $90.9 \%$ & $94 \%$ & $94.2 \%$ \\
\hline PRESENT STUDY & $75 \%$ & $100 \%$ & $100 \%$ & $100 \%$ & $\sim 99 \%$ \\
\hline
\end{tabular}

PPV- Positive predictive value, NPV- Negative predictive value

Table 3: Comparison of study period, number of cases and concordancy with other studies

\begin{tabular}{|l|c|c|c|c|}
\hline Authors & $\begin{array}{c}\text { Study Period } \\
(\text { Years })\end{array}$ & Number of Cases & $\begin{array}{c}\text { Concordant Cases } \\
(\boldsymbol{\%})\end{array}$ & $\begin{array}{c}\text { Discordant Cases } \\
(\boldsymbol{\%})\end{array}$ \\
\hline Shrestha et al (2015) & 5 & 404 & 94.6 & 5.4 \\
\hline Saumya Mishra et al (2015) $^{\mathbf{4}}$ & 2 & 67 & 96.2 & \\
\hline Patil et al (1992) $^{\mathbf{6}}$ & 2 & 100 & 96.9 & 3.8 \\
\hline Chandramouleshwari et al (2016) $^{\mathbf{1 0}}$ & 1 & 51 & 92 & 2 \\
\hline Chbani et al (2012) $^{\mathbf{1 1}}$ & 1 & 261 & 96.2 & \\
\hline PRESENT STUDY & 1 & 45 & 97.7 & 3.8 \\
\hline
\end{tabular}


During the course, of our study we found that the main obstacles encountered, which posed a challenge in the diagnosis of frozen sections were:

- $\quad$ Freezing artefacts

- Cutting artefacts

- Staining artefacts due to the lesser time required for staining

- Lack of adequate history and examination findings in the requisition form

Proper orientation of specimen and clinical details are important to make an accurate diagnosis. A clear communication pertaining to clinical details is important for frozen section diagnosis. Many studies have concluded that disagreements in frozen diagnosis are mostly due to interpretative and sampling errors, followed by sectioning, inadequate history, staining and labelling. CAP specifies that turnaround time in frozen section reporting should be completed within 20 minutes in $90 \%$ or else analysis of outliers should be done.

\section{Conclusion}

Frozen section is a very important diagnostic tool in order to help facilitate adequate surgical procedures. Based on frozen section diagnosis radical surgeries can be changed to conservative surgical procedures and vice versa based on age and therapeutic need of the patient. This diagnostic procedure when conducted with the right indication, with trained technicians and within the limited timeperiod is highly helpful.

Various limitations encountered in our study were due to freezing artefact, inadequate clinical and operative details, lack of orientation and sampling error

Frozen section is subject to various limitations which both surgeon and pathologist should keep in mind when ordering and performing the procedure.

Frozen section diagnosis is a very important and useful method in easy and quick diagnosis for the patient which helps in avoiding repeated procedures provided the surgeon and pathologist act in coordination.

\section{References}

[1] Goss GR. Frozen section: the stat test of clinical pathology? Adv Med Lab. 2001;13:8-12, 82.

[2] Underwood J. Introduction to biopsy interpretation and surgical pathology. 1st ed. London: Springer; 1987.

[3] Gal AA. The centennial anniversary of the frozen section technique at the Mayo Clinic.ArchPathol Lab Med. 2005 Dec; 129(12):1532-5.

[4] Mishra S, Gupta M, Bharat V, Bansal R. Qualitative Comparative Study of Frozen Section with Routine Histological Technique. Nat JLabMed.2016;5(2):44-50

[5] Hatami H, Mohsenifar Z, Alavi SN. The Diagnostic Accuracy of Frozen Section Compared to Permanent Section: A Single Center Study in Iran. Iran J Pathol. 2015; 10(4):295-9.

[6] Patil P, Shukla S, Bhake A, Hiwale K. Accuracy of frozen section analysis in correlation with surgical pathology diagnosis. Int J Res Med Sci. 2015;3(2):399404

[7] Chang J-L, Tseng H-H, Sheu L-F, Lee W-H, Tu Y-C. Diagnostic Accuracy of Frozen Sections in Surgical Pathology-A Retrospective Analysis of 1084 Frozen Sections. Journal of Medical Sciences. 1992; 13(2):13342.

[8] Preeti A, Sameer G, Kulranjan S, et al. Intra-Operative Frozen Sections: Experience at A Tertiary Care Centre. Asian Pac J Cancer Prev. 2016; 17(12):5057-5061.

[9] Shrestha S, Lee MC, Dhakal H, Pun CB, Pradhan M, Basyal [1] $\mathrm{R}$ et al. Comparative study of frozen section diagnoses with histopathology. Postgraduate medical journal of NAMS. 2009;9(2):1-5

[10] Chandramouleeswari K, Yogambal M, Arunalatha P, Bose JC, Rajendran A. Frozen and paraffin sectionsComparative study highlighting the concordance and discordance rates in a tertiary care centre. IOSR J DentalMedSci. 2013; 12(5):26-30.

[11] Chbani L, Mohamed S, Harmouch T, El Fatemi H, Amarti A. Quality assessment of intraoperative frozen sections: An analysis of 261 consecutive cases in a resource limited area: Morocco. Health. 2012;4(7):4335. 